\title{
On The Recently Discovered Correlations Between Gamma-ray And X-ray Properties Of Gamma Ray Bursts
}

\author{
Shlomo Dado ${ }^{1}$ and Arnon Dar ${ }^{1}$
}

\begin{abstract}
Recently, many correlations between the prompt $\gamma$-ray emission properties and the X-ray afterglow properties of gamma-ray bursts (GRBs) have been inferred from a comprehensive analysis of the X-ray light curves of more than 650 GRBs measured with the Swift X-ray telescope (Swift/XRT) during the years 2004-2010. We show that these correlations are predicted by the cannonball (CB) model of GRBs. They result from the dependence of GRB observables on the bulk motion Lorentz factor and viewing angle of the jet of highly relativistic plasmoids (CBs) that produces the observed radiations by interaction with the medium through which it propagates. Moreover, despite their different physical origins, long GRBs (LGRBs) and short hard bursts (SHBs) in the CB model share similar kinematic correlations, which can be combined into triple correlations satisfied by both LGRBs and SHBs.
\end{abstract}

Subject headings: (Stars:) gamma-rays: bursts

\section{Introduction}

As the measurements of the properties of gamma ray bursts (GRBs) and their afterglows (AGs) become more complete and more accurate, new correlations between these properties are being discovered. Such correlations challenge GRB models. Recently, Margutti et al. (2013) reported results from a comprehensive statistical analysis of the X-ray light-curves of more than 650 GRBs (GRBs) measured with the Swift X-ray telescope (Swift/XRT) between 2004 and the end of 2010. In particular, they reported the discovery of new correlations between the properties of the $\gamma$-ray and the X-ray emission in GRBs. In this paper, we show that the newly discovered correlations by Margutti et al. (2013) between the $\gamma$-ray and X-ray properties of GRBs agree well with those predicted by the cannonball (CB) model of GRBs (Dar \& De Rújula 2004, Dado et al. 2009a,b and references therein). Like the previously

\footnotetext{
${ }^{1}$ Physics Department, Technion, Haifa 32000, Israel
} 
well established correlations between GRB observables, in the CB model they all have the same simple kinematic origin - they result from the strong dependence of the observed radiations on the bulk motion Lorentz and Doppler (L\&D) factors of the jet of highly relativistic plasmoids (CBs) that produces the radiations by its interaction with the medium through which it propagates (Dar and De Rújula 2000,2004, Dado et al. 2007, Dado and Dar 2012a).

In this paper, we use the $\mathrm{CB}$ model dependence of the $\gamma$-ray and X-ray properties of GRBs on the Lorentz and Doppler factors to derive the kinematic correlations between them. For completeness, we shall first summarize the origin of the observed radiations in the CB model and the expected correlations among the properties of the prompt $\gamma$-ray emission and among the properties of the X-ray afterglow before proceeding to derive the correlations between them.

\section{Origin of the observed radiations in the $\mathrm{CB}$ model}

In the cannonball (CB) model of GRBs (Dado et al. 2002, Dar and De Rújula 2004, Dado et al. 2009a,b), GRBs and their afterglows are produced by the interaction of bipolar jets of highly relativistic plasmoids $(\mathrm{CBs})(\gamma \gg 1)$ of ordinary matter with the radiation and matter along their trajectory (Shaviv and Dar 1995, Dar 1998). Such jetted CBs are presumably ejected in accretion episodes on the newly formed compact stellar object in core-collapse supernova (SN) explosions (Dar et al. 1992, Dar and Plaga 1999, Dar and De Rújula 2000), in merger of compact objects in close binary systems (Goodman et al. 1987, Shaviv and Dar 1995) and in phase transitions in compact stars (Dar 1998, Dar and De Rújula 2000, Dado et al. 2009b). For instance, in long GRBs produced in Type Ic supernova explosions it is hypothesized that an accretion disk or a torus is produced around the newly formed compact object, either by stellar material originally close to the surface of the imploding core and left behind by the explosion-generating outgoing shock, or by more distant stellar matter falling back after its passage (Dar and De Rújula 2000, 2004). As observed in microquasars, each time part of the accretion disk falls abruptly onto the compact object, two CBs made of ordinary-matter plasma are emitted in opposite directions along the rotation axis from where matter has already fallen back onto the compact object due to lack of rotational support. The prompt $\gamma$-ray pulses and early-time X-ray flares are dominated by inverse Compton scattering (ICS) of glory photons - a light halo surrounding the progenitor star that was formed by stellar light scattered from the pre-supernova ejecta/wind blown from the progenitor star - by the CBs' electrons. The ICS is overtaken by synchrotron radiation (SR) when the CB enters the pre-supernova wind/ejecta of the progenitor star. The fast decline of the prompt emission with a fast spectral softening is overtaken (see, e.g., Dado et 
al. 2007, 2009a) by a broad-band SR afterglow with a much harder spectral index $\beta_{X} \simeq 1$, which stays constant in the X-ray band, when the CB propagates in the wind and interstellar (ISM) environment. ICS of the SR produces the emission of very high energy photons during the early time optical/NIR emission and the broad band afterglow (Dado and Dar 2009).

\section{Origin of correlations in the $\mathrm{CB}$ model}

Detailed discussion of the origin of correlations between GRB properties can be found in Dar and De Rújula 2000, 2004, Dado et al. 2007, and Dado and Dar 2012a. In short, GRBs are not standard candles because of the diversity of their central engines and environments. But, because of the large bulk motion Lorentz factor $\gamma$ of the jet of CBs, their emitted radiation at redshift $z$, which is observed at a small angle $\theta$ relative to the direction of the jet, is boosted by a large Doppler factor $\delta=1 / \gamma(1-\beta \cos \theta)$ and collimated through relativistic beaming by a factor $\delta^{2}$. In the CB model, the large Doppler boosting, relativistic beaming and time aberration dominate the GRB properties and produce correlations between GRB observables, which depend on them (Dar and De Rùjula 2000, 2004, Dado and Dar 2012a), despite their dependence on the CBs' intrinsic properties (rest frame properties indicated from now on by a prime) and on the environment along their trajectories (which produce a significant spread around these simple kinematic correlations).

\section{Correlations among and between $\gamma$-ray and X-ray properties}

\subsection{Prompt emission}

Prompt $\gamma$-rays: In the $\mathrm{CB}$ model, the prompt emission is a sum of individual pulses. For standard candle GRBs, in each pulse the peak energy $E_{p}$ of the time-integrated spectral energy flux, the total gamma-ray energy emission under the assumption of isotropic emission $E_{i s o}$, and the peak luminosity $L_{p}$ satisfy $E_{p}^{\prime} \propto \gamma_{0} \delta_{0}, E_{i s o} \propto \gamma_{0} \delta_{0}^{3}$ and $L_{p} \propto \gamma_{0}^{2} \delta_{0}^{4}$, respectively. Consequently, each individual pulse in a GRB is predicted to satisfy the triple correlation, $L_{p} \propto E_{p}^{\prime} E_{i s o}$. The most probable viewing angle of GBs is $\theta \approx 1 / \gamma_{0}$ yielding $\delta_{0} \approx \gamma_{0}$ and the approximate binary power-law correlations $(1+z) E_{p} \propto\left[E_{i s o}\right]^{0.5},(1+z) E_{p} \propto\left[L_{p}\right]^{0.33}$, and $L_{p} \propto\left[E_{i s o}\right]^{1.5}$, respectively. For XRFs that in the CB model are GRBs viewed far offaxis, i.e., $\theta \gg 1 / \gamma$, the $\mathrm{CB}$ model yields $(1+z) E_{p} \propto\left[E_{i s o}\right]^{1 / 3},(1+z) E_{p} \propto\left[L_{p}\right]^{0.25}$, and $L_{p} \propto\left[E_{i s o}\right]^{4 / 3}$.

For the time-integrated $\gamma$-ray emission in multi-peak GRBs, the CB model predicts for each pulse and for the entire GRB, roughly the same power-law index $\approx 0.5$ for the $E_{p}^{\prime}-E_{\gamma, \text { iso }}$ 
correlation. The peak value of the luminosity integrated over a fixed observer time step, e.g., over 1 second, satisfies $L_{p, s} \propto \gamma_{0} \delta_{0}^{3}$ and consequently $E_{i s o} \propto L_{p, s}$ and $(1+z) E_{p} \propto\left[L_{p, s}\right]^{0.5}$. The above CB model correlations, which were predicted (Dar \& De Rújula 2000, 2004) before their empirical discovery (Amati et al. 2002; Yonetoku et al. 2004), are well satisfied by GRBs, as shown in Figs. 1-3.

Prompt X-rays: In the CB model, the prompt emission is dominated by ICS of thin thermal bremsstrhalung (Dar \& De Rújula 2004) whose time-integrated spectrum is given roughly by an exponentially cut-off power-law (CPL), $\int E(d n / d E) d t \sim E^{1-\alpha} e^{-E / E_{c}}$, where $\alpha \approx 1$, and then the $E_{p}$, the peak energy of $E^{2} \int(d n / d E) d t$ is roughly the "cut-off" energy $E_{c}$. Consequently, for the X-ray band, which is usually well below $E_{p}$, the emitted prompt $\mathrm{X}$-ray energy, within a band width $\Delta E$ is given by

$$
E_{1, X} \approx \frac{\Delta E}{E_{p}} E_{\gamma, i s o}
$$

where $E_{\gamma, i s o}=E_{i s o}$ and $E_{1, X}$ are, respectively, the prompt gamma ray and X-ray energies emitted under the assumption of isotropic emission. Using the CB model relations (Dar \& De Rújula 2000,2004), $E_{p}^{\prime} \propto \gamma_{0} \delta_{0}$ and $E_{i s o} \propto \gamma_{0} \delta_{0}^{3}$, one obtains $E_{1, X} \propto \delta_{0}^{2}$.

If the X-ray energy $E_{1, X}$ emitted during the first phase of the X-ray light-curve measured with Swift is proportional to the prompt X-ray emission, then Eq. (1) implies that $E_{1, X} \propto$ $\left[E_{\gamma, i s o}\right]^{0.58 \pm 0.08}$, where the exact power-law index depends on the mixure of ordinary GRBs and XRFs in the observed sample. This predicted index is in agreement with the correlation $E_{1, X} \propto\left[E_{i s o}\right]^{0.56 \pm 0.04}$ reported in Margutti et al. 2013.

\subsection{The X-ray afterglow}

In the CB model, the X-ray afterglow is dominated by synchrotron radiation (SR), and begins when the (merged) CBs enter the circumburst wind, which have been blown by the progenitor star sometime before the SN explosion. The spectral energy density of the unabsorbed X-ray afterglow of a single CB has the form (see, e.g., Eq. (26) in Dado et al. 2009a),

$$
F_{\nu} \propto n^{\left(\beta_{X}+1\right) / 2}[\gamma(t)]^{3 \beta_{X}-1}[\delta(t)]^{\beta_{X}+3} \nu^{-\beta_{X}},
$$

where $n$ is the baryon density of the medium, and $\beta_{X}+1=\Gamma_{X}$ is the photon spectral index of the emitted (unabsorbed) radiation. For $\gamma^{2} \gg 1$ and $\theta^{2} \ll 1, \delta \approx 2 \gamma /\left(1+\gamma^{2} \theta^{2}\right.$ ) to an excellent approximation. The X-ray band is well above the break frequency, where typically $\Gamma_{X} \approx 2$, i.e., $\beta_{X} \simeq 1$, and Eq. (2) yields $F_{\nu} \propto n[\gamma]^{2}[\delta]^{4} \nu^{-1}$. For a "shot-gun" configuration 
of CBs, Eq. (2) yields (Dado and Dar 2012b)

$$
F_{\nu} \propto n^{\left(\beta_{X}+1\right) / 2}[\gamma(t)]^{4 \beta_{X}} \nu^{-\beta_{X}}
$$

which reduces to $F_{\nu} \propto n[\gamma(t)]^{4} \nu^{-1}$ for $\beta_{X} \simeq 1$.

\subsection{The early-time and break-time luminosities of canonical AGs}

The intercepted ISM particles that are swept into the CB decelerate its motion. For a CB of a baryon number $N_{B}$, a radius $R$ and an initial Lorentz factor $\gamma_{0}=\gamma(0) \gg 1$, which propagates in an ISM of a constant density $n$, relativistic energy-momentum conservation yields the deceleration law (Dado et al. 2009b and references therein)

$$
\gamma(t)=\frac{\gamma_{0}}{\left[\sqrt{\left(1+\theta^{2} \gamma_{0}^{2}\right)^{2}+t / t_{0}}-\theta^{2} \gamma_{0}^{2}\right]^{1 / 2}},
$$

where $t_{0}=(1+z) N_{\mathrm{B}} / 8 c n \pi R^{2} \gamma_{0}^{3}$.

As long as $t_{\sim}^{<} t_{b}=\left(1+\gamma_{0}^{2} \theta^{2}\right)^{2} t_{0}, \gamma(t)$ and $\delta(t)$ change rather slowly with $t$, which generates the plateau phase of $F_{\nu}(t)$ of canonical X-ray AGs that was predicted by the CB model (see, e.g., Dado et al. 2002, Figs. 27-33) and later observed with Swift (Nousek et al. 2006, Panaitescu et al. 2006, Zhang et al. 2006). For $\beta_{X} \simeq 1$, the X-ray luminosity at the beginning time $t_{i}$ of the plateau phase that follows from Eq. (2) is given by $L_{X}\left(t_{i}\right) \propto \gamma_{0}^{2} \delta_{0}^{4}$. From Eq. (4) it follows that for $t \gg t_{b}, \gamma(t) \rightarrow \gamma_{0}\left(t / t_{b}\right)^{-1 / 4},[\gamma(t) \theta]^{2}$ becomes $\ll 1$ and $\delta \approx 2 \gamma(t)$, which result in a late-time power-law decay

$$
F_{\nu}(t) \propto\left[\gamma_{0}\right]^{4 \beta_{X}+2}\left(t / t_{b}\right)^{-\beta_{X}-1 / 2}
$$

Hence, the dependence on the L\&D factors of the break-time is $t_{b}^{\prime} \propto 1 / \gamma_{0} \delta_{0}^{2}$, which yields the triple correlation

$$
t_{b}^{\prime} \propto 1 /\left[E_{p}^{\prime} E_{i s o}\right]^{1 / 2} .
$$

Then, the substitution $t_{b}=(1+z) t_{b}^{\prime}$ and $E_{p}^{\prime} \propto\left[E_{i s o}\right]^{1 / 2}$ yields the approximate pair correlations

$$
t_{b} /(1+z) \propto\left[E_{i s o}\right]^{-0.75} \propto\left[E_{p}^{\prime}\right]^{-1.5} .
$$

Note that the predicted late-time $\left(t^{\prime} \gg t_{b}^{\prime}\right)$ behaviour of the X-ray luminosity at a fixed $t^{\prime}$,

$$
L\left(t^{\prime}\right) \propto \gamma_{0}^{1.5} t^{\prime-1.5} \propto\left[E_{i s o}\right]^{0.5} t^{\prime-1.5}
$$

is in agreement with the observed behaviour $L_{X}(11 h) \propto\left[E_{\text {iso }}\right]^{0.50}$ (Margutti et al. 2013). 
Note also that for a 'shotgun' configuration of CBs, $L_{X}\left(t_{i}\right) \propto \gamma_{0}^{4 \beta_{X}}$ and $L_{X}\left(t \gg t_{b}\right) \propto$ $\gamma_{0}^{4 \beta_{X}}\left(t / t_{b}\right)^{-\beta_{X}}$ (Dado \& Dar 2012b), and the late-time power-law behaviour of $L_{X}$ extrapolated back to $t=t_{b}$ yields $L_{X}\left(t_{b}\right) \propto \gamma_{0}^{4 \beta_{X}}$. Hence, for $\beta \simeq 1$, roughly $L_{X}\left(t_{b}\right) \propto L_{X}\left(t_{i}\right) \propto \gamma_{0}^{5 \pm 1}$.

The CB model (achromatic) break-time parameter $t_{b}$, however, is not the same as the chromatic break-time parameter of the heuristic smooth broken power-law function used by Margutti et al. (2013) to parametrize canonical AGs. Consequently, the correlations satisfied by the $\mathrm{CB}$ model break-time of the X-ray AG may differ slightly from those inferred from the phenomenological broken power-law fits reported in Margutti et al. 2013.

\section{4. $\quad E_{2, X}$ in canonical GRBs}

Assuming isotropic emission in the GRB rest frame at redshift $z$ (luminosity distance $d_{L}$ ), the total emitted energy during the afterglow phase in the rest frame X-ray band $\left[\nu_{1}^{\prime}, \nu_{2}^{\prime}\right]$ is given by

$$
E_{2, X}=\frac{4 \pi d_{L}^{2}}{(1+z)} \iint F_{\nu}(t) d t d \nu
$$

where the $\nu$ integration is from $\nu_{1}=\nu_{1}^{\prime} /(1+z)$ to $\left.\nu_{2}=\nu_{2}^{\prime} /(1+z)\right]$. Since $F_{\nu} \propto \nu^{-\beta_{X}} \sim \nu^{-1}$, the $\nu$ integration is practically independent of redshift. Moreover, Eq. (4) can be used to convert the time integration in Eq. (9) to integration over $\gamma$, yielding $E_{2, X} \propto \gamma_{0}^{2} \delta_{0}$ for the total energy emitted in the $0.3-30 \mathrm{keV}$ X-ray band during the afterglow phase, assuming isotropic emission.

\section{Comparison with observations}

\subsection{Binary correlations}

Table 1 Summarizes the dependence in the CB model of $\gamma$-ray and X-ray properties of GRBs on the bulk motion L\&D factors of the CBs, which was detailed in section 4. In Table 1, we have used the notation $E_{p}^{\prime}$ and $L_{i s o}$ for the rest frame peak gamma ray energy and the mean luminosity during T90, respectively, of the prompt $\gamma$-ray emission.

Table 2 presents a comparison between the best fit indices of the 21 observed power-law correlations among seven chosen observables and those predicted by the cannonball model. The predicted indices are the arithmetic mean of the typical cases $\theta \gamma_{0} \approx 1$ and $\theta^{2} \gamma_{0}^{2} \gg 1$.

Table 3 summarizes the observed correlations between $E_{p}^{\prime}, E_{\text {iso }}$ and $t_{b}$ and the power-law correlation indices expected in the cannonball model and in the collimated fireball model. 
Figs. 1-3 present our best fitted power-laws for the $E_{p}^{\prime}-E_{i s o}, E_{p}^{\prime}-L_{i s o}$, and $L_{i s o}-E_{i s o}$ correlations for a sample of 96 GRBs with known redshift, which was compiled by Yonetoku et al. (2010). Using essentially the method advocated by D'Agostini (2005), we obtained the best fit correlations $(1+z) E_{p} \propto\left[E_{i s o}\right]^{0.529},(1+z) E_{p} \propto\left[L_{i s o}\right]^{0.532}$, and $L_{p, \gamma} \propto\left[E_{i s o}\right]^{1.13}$ in good agreement with those predicted by the CB model. Note that the correlations satisfied by $E_{p}$ imply that $E_{i s o} \propto\left[L_{i s o}\right]^{1.01}$. The $\sim 10 \%$ difference in the power-law index of the $E_{i s o}-L_{i s o}$ correlation probably provides a realistic estimate of the accuracy of the power-law indices extracted from the observational data.

Fig. 4 compares the triple $t_{b}^{\prime}-E_{p}^{\prime}-E_{\text {iso }}$ correlation predicted by the CB model (Eq. 6) and the observed correlation in 68 Swift GRBs (Evans et al. 2009) from the above GRB sample, which have a good Swift/XRT temporal coverage of their X-ray afterglow during the first day (or more) following the prompt emission phase and have no superimposed flares. In this sample, the X-ray afterglow of 54 GRBs clearly shows a break and no afterglow-break was observed in $14 \mathrm{GRBs}$. In order not to bias the values of $t_{b}, E_{p}$, and $E_{\text {iso }}$ by the CB model fits, the break times were taken to be the times of the first break with $\alpha\left(t<t_{b}\right)<\alpha\left(t>t_{b}\right)$ obtained from the broken power-law fit to the GRB X-ray afterwglow measured with the Swift/XRT and reported in the Leicester XRT GRB Catalogue (Evans et al. 2009). The Spearman rank (correlation coefficient) of the $t_{b}^{\prime}-\left(E_{p}^{\prime} E_{i s o}\right)$ correlation is $r=-0.65$ with a chance probabilities less than $2.6 \times 10^{-9}$. The best fit triple correlation $t_{b}^{\prime} \propto 1 /\left[E_{p}^{\prime} E_{i s o}\right]^{p}$ yields $p=-0.58$

The approximate binary correlations $t_{b}^{\prime}-E_{i s o}$ and $t_{b}^{\prime}-E_{p}^{\prime}$ that were obtained by substitution of the CB model predicted correlation $E_{p}^{\prime} \propto\left[E_{i s o}\right]^{1 / 2}$ in the triple correlation $t_{b}^{\prime}-E_{p}^{\prime}-E_{i s o}$ (Eq. 6), are compared with the observational data in Figs. 5 and 6, respectively. GRB 980425 was excluded from the GRB sample because it is an outlier with respect to the binary $E_{p}^{\prime}-E_{i s o}$ correlation (as expected in the CB model). The Spearman ranks of the observed $t_{b} /(1+z)-E_{\text {iso }}$ and $t_{b} /(1+z)-(1+z) E_{p}$ correlations are -0.49 and -0.63 with chance probabilities less than $4.5 \times 10^{-4}$ and $1.0 \times 10^{-6}$, respectively. As expected in the CB model, they are larger than that of the $t_{b}^{\prime}-\left(E_{p}^{\prime} E_{i s o}\right)$ correlation. The best fit power-law indices of the $t_{b}^{\prime}-E_{i s o}$ and $t_{b}^{\prime}-E_{p}^{\prime}$ correlations are $p=-0.70 \pm 0.06$ and $p=-1.64 \pm 0.04$, respectively, consistent with their predicted values by the $\mathrm{CB}$ model, -0.75 and -1.50 , respectively.

The best fit power-law indices $p=0.54 \pm 0.01, p=-1.61 \pm 0.04$, and $p=-0.70 \pm 0.06$ of the observed $E_{p}^{\prime}-E_{i s o}, t_{b}^{\prime}-E_{p}^{\prime}$ and $t_{b}^{\prime}-E_{\text {iso }}$ power-law correlations, respectively, are at odds with the values $1,-1$, and -1 , respectively, expected in the conical fireball model.

Table 2 compares the approximate power-law correlations between the $\gamma$-ray and Xray propreties of long GRBs that are expected in the CB model (upper rows) from their dependence on the L\&D factors and those extracted in the limit of small dispersions from 
the correlations reported in Margutti et al. 2013 (lower rows). As can be seen from Table 2, the correlations predicted by the CB model agree well with those inferred by Margutti for LGRBs.

\subsection{The triple correlation $E_{X, i s o}-E_{\gamma, i s o}-E_{p}^{\prime}$}

A triple correlation $E_{X, \text { iso }} \propto\left[E_{\gamma, i s o}\right]^{1.06 \pm 0.06} /\left[E_{p}^{\prime}\right]^{0.74 \pm 0.10}$ was found by Bernardini et al. (2012) to be well satisfied by both long and short GRBs. This correlation was updated in Margutti et al. (2013) to be $E_{X, i s o} \propto\left[E_{\gamma, i s o}\right]^{1.00 \pm 0.06} /\left[E_{p}^{\prime}\right]^{0.60 \pm 0.10}$. In the CB model, such triple correlations that unite LGRBs and SHBs are a simple combination of corresponding binary power-law correlations of kinematic origin satisfied by LGRBs and SHBs (with the same index but different normalization). In particular, the above triple correlation is a simple consequence of the correlation $E_{p}^{\prime} \propto\left[E_{\gamma, i s o}\right]^{p}$ (shown in Fig. 1 for LGRBs with the current best fit value $p=0.54$ ) that was predicted by the CB model to be satisfied both by LGRBs (e.g., Dar and De Rújula 2000,2004) and by SHBs (Dado et al. 2009b, Fig. 5) and the pair correlation $E_{X, i s o} \propto\left[E_{\gamma, i s o}\right]^{0.67 \pm 0.01}$ that was discovered by Margutti et al. (2013) for LGRBs. To see that, let us rewrite the $E_{X, i s o}-E_{\gamma, i s o}$ correlation that was found by Margutti et al. (2013) for LGRBs in the form

$$
E_{X, i s o} \propto\left[E_{\gamma i s o}\right]^{0.67} \frac{\left[E_{\gamma i s o}\right]^{m}}{\left[E_{p}^{\prime}\right]^{m / p}},
$$

where the second factor on the right hand side (RHS) is a constant. For the triple correlation reported by Margutti et al. (2013), their best fit implies $m=0.33 \pm 0.06$ and $m / p=$ $0.60 \pm 0.10$, which yield $p=(0.33 \pm 0.06) /(0.60 \pm 0.10)=0.55 \pm 0.07$. The best fit values reported by Bernardini et al. (2012) yield $m=0.39 \pm 0.06$ and $m / p=0.74 \pm 0.10$, which implies $p=(0.39 \pm 0.06) /(0.74 \pm 0.10)=0.527 \pm 0.05$. Both values are consistent with the CB model prediction $p \approx 0.5$ and with $p=0.526$ obtained from the best fit shown in Fig. 1 . For LGRBs, the triple correlation as written in Eq. (10) is independent of the choice of an $m$ value. However, in the $\mathrm{CB}$ model the power-law indices of kinematic correlations are common to SHBs and LGRBs. Thus, the value of $m$ can be adjusted such that the triple correlation in LGRBs is satisfied also by SHBs.

\subsection{The triple correlation $t_{b}^{\prime}-E_{p}^{\prime}-E_{\gamma, i s o}$}

As explained in section 3, the strong dependence of GRB observables on both the Lorentz factor and the Doppler factor of the jetted CBs yields triple-correlations among these observables, which can be reduced to binary correlations only with additional assumptions. 
We demonstrate this for an important case - the triple correlation $t_{b}^{\prime}-E_{p}^{\prime}-E_{\gamma, i s o}$ as summarized in Eq. (6), which is based on the decelaration origin of the break in the canonical afterglow of the CB model. In Fig. 4, this correlation is compared with observations of 68 Swift GRBs/XRFs with known redshift, good temporal coverage of their X-ray afterglow during the first day (or more) following the prompt emission phase, and well measured $E_{p}$ and $E_{i s o}$ with Konus-WIND and/or Fermi GBM. In order not to bias the values of $t_{b}^{\prime}, E_{p}^{\prime}$ and $E_{\text {iso }}$ by $\mathrm{CB}$ model fits, the break times were taken to be the times of the first break with $\alpha\left(t<t_{b}\right)<\alpha\left(t>t_{b}\right)$ obtained from the broken power-law fits to the GRB X-ray afterglow measured with the Swift/XRT and reported in the Leicester XRT GRB Catalogue (Evans et al. 2009). The values of $E_{p, \gamma}$ and $E_{\gamma, i s o}$ were adopted from communications of the KonusWind and Fermi GBM collaborations to the GCN Circulars Archive (Barthelmy 1997), and from publications by Amati et al. (2007, 2008 ), Yonetoku et al. (2010), Gruber et al. (2011), Nava et al. (2012), and D'Avanzo et al. (2012). As shown in Fig. 4, the triple correlations predicted by the CB model is well satisfied by the observational data; the best fit power-law $t_{b}^{\prime} \propto\left[E_{p}^{\prime} E_{\gamma, i s o}\right]^{p}$ yields $p=-0.58 \pm 0.04$ in good agreement with the predicted power-law index $p=-1 / 2$.

The binary correlations $t_{b} /(1+z) \propto\left[E_{i s o}\right]^{-0.75} \propto\left[E_{p}^{\prime}\right]^{-1.5}$ that were obtained by substituting the $\mathrm{CB}$ model correlation $E_{p}^{\prime} \propto E_{i s o}^{1 / 2}$ in the triple correlation (Eq. 6) are in good agrement with the the best fit power-law correlations shown in Figs. 5 and 6, with power-law indices $-0.69 \pm 0.06$ and $-1.62 \pm 0.04$, respectively.

In particular, the break-time correlations imply that GRBs with very large values of $E_{i s o}$ and $E_{p}^{\prime}$ have a small $t_{b}^{\prime}$ value, which, probably, is hidden under the tail of the prompt emission or precedes the start of the XRT observations (Dado et al. 2007). Indeed, the X-ray afterglow of all the GRBs in our sample, which have large values of $E_{i s o}$ and $E_{p}^{\prime}$, such as 061007, 080319B and 130427A, have a power-law decline consistent with the post break power-law decline predicted by the CB model (see, e.g., Dado et al. 2007, 2009a). For such GRBs, the observations provide only upper bounds on the break time of their X-ray afterglow, which are indicated by down arrows in Figs. 4-6.

\section{Discussion}

A major breakthrough in the study of gamma ray bursts (GRBs) was the discovery of their X-ray afterglows by the Beppo-SAX satellite (Costa et al. 1997) that allowed their arcminute localization and consequently the discovery of their longer wave-length afterglows (van Paradijs et al. 1997, Frail and Kulkarni 1997), which were predicted (Paczynski and Roads 1993, Katz 1994, Meszaros and Rees 1997) by the fireball model (FB) of 
GRBs (Paczynski 1986, Goodman 1986). Consequently, the fireball model was widely accepted as the correct model of GRBs and their afterglows (e.g., Meszaros 2002, Zhang and Meszaros 2004, Zhang 2007). The rich data on GRBs and their afterglows obtained in recent years with the Swift and Fermi satellites, complemented by data from ground-based rapid response telescopes and large follow-up telescopes, have, however, challenged this prevailing view (e.g., Covino et al. 2006, Curran et al. 2006, Burrows and Racusin 2006, Kumar et al. 2007, Zhang et al. 2007, Liang et al. 2008, Godet \& Mochkovitch 2011, Margutti et al. 2013, and references therein).

In contrast, the cannonball model $(\mathrm{CB})$ of GRBs has been very successful in predicting the general properties of the prompt emission and afterglows of both long and short GRBs and in reproducing their detailed light-curves (e.g., Dado et al. 2009a,b and references therein). This success was despite the apparently different origins and environments of long GRBs and SHBs. But, it involved an adjustment of free parameters for each GRB, which could have made one wonder whether the agreement between theory and observations was due to the flexibility of the model rather than its validity. Many properties of GRBs, correlations among them, and closure relations that are predicted by the CB model, however, do not involve adjustable parameters and thus enable more stringent tests of the model. So far the CB model was able to predict correctly all the main established correlations among GRB observables (see, e.g., Dar and De Rújula 2000,2004, Dado and Dar 2012a,b and references therein) including the newly discovered correlations (Liang et al. 2010) among and between the prompt $\gamma$-ray and optical properties of GRBs (e.g., Dado and Dar 2012a and references therein). In this paper, we have shown that the correlations that were discovered recently between the $\gamma$-ray and X-ray properties of GRBs (Margutti et al. 2013, Bernardini et al. 2012) are correctly predicted by the CB model. Moreover, we have also shown that the triple correlation $E_{X, i s o}-E_{\gamma, \text { iso }}-E_{p}^{\prime}$ that is satisfied by both LGRBs and SHBs (Bernardini et al. 2012, Margutti et al. 2013) probably is a simple consequence of the fact that the binary power-law correlations $E_{X, i s o}-E_{\gamma, i s o}$ and $E_{p}^{\prime}-E_{\gamma, i s o}$ are satisfied by both LGRBs and SHBs with roughly the same power-law index and different normalizations, as expected in the CB model.

Finally, we note that, in contrast to the cannonball model, the conical fireball model has not been able to explain the observed correlations between the prompt and afterglow emissions, in particular those which involve the afterglow break-time, as was shown in detail in this paper.

Acknowledgment: We thank Raffaela Margutti and an anonymous referee for useful comments, and David Gruber for useful communications. 


\section{REFERENCES}

Amati, L., et al. 2002, A\&A, 390, 81

Amati, L., et al. 2007, A\&A, 463, 913A

Amati, L., et al. 2008, MNRAS, 391, 577

Barthelmy, S., 1997, GCN Circulars Archive, http://gcn.gsfc.nasa.gov/gcn_main.html

Bernardini, M. G., et al. 2012, arXiv:1203.1060

Burrows, D. N. \& Racusin, J. 2006, Nuovo Cim. B121, 1273

Costa, E., et al. 1997, Nature, 387, 783

Covino, S., et al. 2006, Il Nuovo Cimento B, 121, 1171

Curran, P. A., et al. 2006, Nuovo Cim. B121, 1461

Dado, S. \& Dar, A. 2009, arXiv:0910.0687

Dado, S. \& Dar, A. 2012a, ApJ, 749, 100

Dado, S. \& Dar, A., 2012b, ApJ, 761, 148

Dado, S., Dar, A. \& De Rújula, A. 2002, A\&A, 388, 1079

Dado, S., Dar, A. \& De Rújula, A., 2007, ApJ, 663, 400

Dado, S., Dar, A. \& De Rújula, A. 2009a, ApJ, 696, 994

Dado, S., Dar A., De Rújula A., 2009b, ApJ, 693, 311

D’Agostini, G., 2005, arXiv:physics/0511182

Dar, A., 1998, ApJ, 500, L93

Dar, A. \& De Rújula A., 2000, arXiv:astro-ph/0012227

Dar, A. \& De Rújula, A. 2004, Phys. Rep. 405, 203

Dar, A., Kozlovsky, B., Nussinov, S. \& Ramaty R., 1992, ApJ, 388, 164

Dar, A. \& Plaga, R., 1999, A\&A, 349, 259

D'Avanzo, P., et al. 2012, arXiv:1206.2357 
Evans, P. A., et al. 2009, MNRAS, 397, 1177

Frail, D. A. \& Kulkarni, S. R., 1997, IAU Circ. No. 662

Godet \& Mochkovitch, 2011, Compte Rendu Physque, 12, 276

Goodman, J., 1986 ApJ, 308, L47

Goodman, J., Dar, A. \& Nussinov, S. 1987, ApJ, 314, L7

Gruber, D., et al. 2011, A\&A, 531A, 20

Katz, J., 1994, ApJ, 432, L107

Kumar, P., et al. 2007, MNRAS, 376, L57

Liang, E. W., et al. 2008, ApJ, 675, L528

Liang, E. W., et al. 2010, ApJ, 725, 2209

Margutti, R., et al. 2013, MNRAS, 428, 729

Meszaros, P., 2002, ARAA, 40, 137

Meszaros, P. \& Rees M. J. 1997, ApJ, 476, 232

Nava, L., et al. 2012, MNRAS, 421, 1256

Nousek, J. A., et al. 2006, ApJ, 642, 389

Paczynski, B., 1986, ApJ, 308, L43

Paczynski, B. \& Rhoads, J. E., 1993, ApJ, 418, L5

Panaitescu, A., et al. 2006, MNRAS, 366, 1357

Shaviv, N. J. \& Dar, A., 1995, ApJ, 447, 863

Yonetoku, D., et al. 2004, ApJ, 609, 935

Yonetoku, D., et al. 2010, PASJ, 62, 1495

van Paradijs, J., et al. 1997, Nature, 386, 686

Zhang, B., et al. 2006, ApJ, 642,

Zhang, B. 2007, ChjAA, 7, 1 
Zhang, B-B., Liang, E-W. \& Zhang, B. 2007, ApJ, 666, 1002

Zhang, B. \& Meszaros, P., 2004, Int. J. Mod. Phys. A, 19, 2385

This preprint was prepared with the AAS LATEX macros v5.2.

Table 1. The dependence on Lorentz and Doppler factors of LGRB properties in the CB model

\begin{tabular}{ccccccccc}
\hline \hline property: & $E_{1, X}$ & $E_{2, X}$ & $L_{X}\left(t_{i}\right)$ & $L_{X}\left(t_{b}^{\prime}\right)$ & $t_{b}^{\prime}$ & $E_{\gamma, i s o}$ & $E_{p}^{\prime}$ & $L_{p, s}>$ \\
\hline propto: & $\delta_{0}^{2}$ & $\gamma_{0}^{2} \delta_{0}$ & $\gamma_{0}^{2} \delta_{0}^{4}$ & $\gamma_{0}^{3} \delta_{0}^{3}$ & $1 / \gamma_{0} \delta_{0}^{2}$ & $\gamma_{0} \delta_{0}^{3}$ & $\gamma_{0} \delta_{0}$ & $\gamma_{0} \delta_{0}^{3}$ \\
\hline \hline
\end{tabular}


Table 2. Comparison between the power-law index $m$ of the correlations $Y \propto X^{m}$ predicted by the CB model (upper rows) for various pairs $(X, Y)$ of properties of long duration GRBs and those obtained (lower rows) by Margutti et al. (2013) from a comprehensive analysis of the X-ray light curves of more than 650 GRBs measured with the Swift X-ray telescope (XRT) during the years 2004-2010. The correlations satisfied by the afterglow break-time were inferred from the Swift/XRT light-curves (Evans et al. 2009) by the authors of the present paper

\begin{tabular}{clllllll}
\hline \hline $\mathrm{Y}$ & $E_{2, X}$ & $L_{X}\left(t_{i}^{\prime}\right)$ & $L_{X}\left(t_{b}^{\prime}\right)$ & $t_{b}^{\prime}$ & $E_{i s o}$ & $E_{p}^{\prime}$ & $L_{p, s}$ \\
\hline$E_{2, X}$ & 1 & 0.50 & 0.50 & -1 & 0.71 & 1.50 & 0.60 \\
& 1 & 0.46 & 0.50 & -0.99 & 0.74 & 1.48 & 0.60 \\
\hline$L_{X}\left(t_{i}^{\prime}\right)$ & 2 & 1 & 1 & -2.0 & 1.5 & 3 & 1.5 \\
& 2.17 & 1 & 1.09 & -1.52 & 1.52 & 2.71 & 1.31 \\
\hline$L_{X}\left(t_{b}^{\prime}\right)$ & 2 & 1 & 1 & -2.0 & 1.50 & 3 & 1.00 \\
& 2 & 0.92 & 1 & -1.71 & 1.06 & 2.02 & 1.06 \\
\hline$t_{b}^{\prime}$ & -1. & -0.50 & -0.50 & 1 & -0.75 & -1.50 & -0.75 \\
& -1.07 & -0.66 & -0.63 & 1 & -0.70 & -1.61 & -0.61 \\
\hline$E_{i s o}$ & 1.41 & 0.67 & 0.67 & -1.33 & 1 & 2 & 1 \\
& 1.35 & 0.66 & 0.63 & -1.43 & 1 & 1.90 & 0.88 \\
\hline$E_{p}^{\prime}$ & 0.67 & 0.33 & 0.33 & -0.67 & 0.50 & 1 & 0.50 \\
& 0.78 & 0.37 & 0.36 & -0.62 & 0.53 & 1 & 0.52 \\
\hline$L_{p, s}$ & 1.67 & 0.67 & 1.00 & -1.33 & 1. & 2.0 & 1 \\
& 1.59 & 0.76 & 0.86 & -1.43 & 1.13 & 2.14 & 1 \\
\hline \hline
\end{tabular}

Table 3. Summary of the observed power-law correlations between $E_{p}^{\prime}, E_{\text {iso }}$, and $t_{b}^{\prime}$ and their power-law indices expected in the CB model and in the collimated FB model. $\rho$ is the Spearman rank (corrrelation coefficient), $P(\rho)$ is the probability to obtain by chance a correlation coefficient larger than $|\rho|$, and $p$ is the power-law index of the correlation.

\begin{tabular}{cclccc}
\hline \hline Correlation & $\rho$ & $P(\rho)$ & $p(o b s)$ & $p(C B)$ & $p(F B)$ \\
\hline$E_{p}^{\prime}-E_{\text {iso }}$ & +0.87 & $\approx 0$ & $+0.54 \pm 0.01$ & +0.50 & +1 \\
$t_{b}^{\prime}-\left(E_{p}^{\prime} E_{\text {iso }}\right)$ & -0.70 & $\approx 2.6 \times 10^{-9}$ & $-0.58 \pm 0.04$ & -0.50 & \\
$t_{b}^{\prime}-E_{p}^{\prime}$ & -0.63 & $\approx 1.0 \times 10^{-6}$ & $-1.62 \pm 0.04$ & -1.50 & -1.0 \\
$t_{b}^{\prime}-E_{\text {iso }}$ & -0.49 & $\approx 4.5 \times 10^{-4}$ & $-0.69 \pm 0.06$ & -0.750 & -1.0 \\
\hline \hline
\end{tabular}




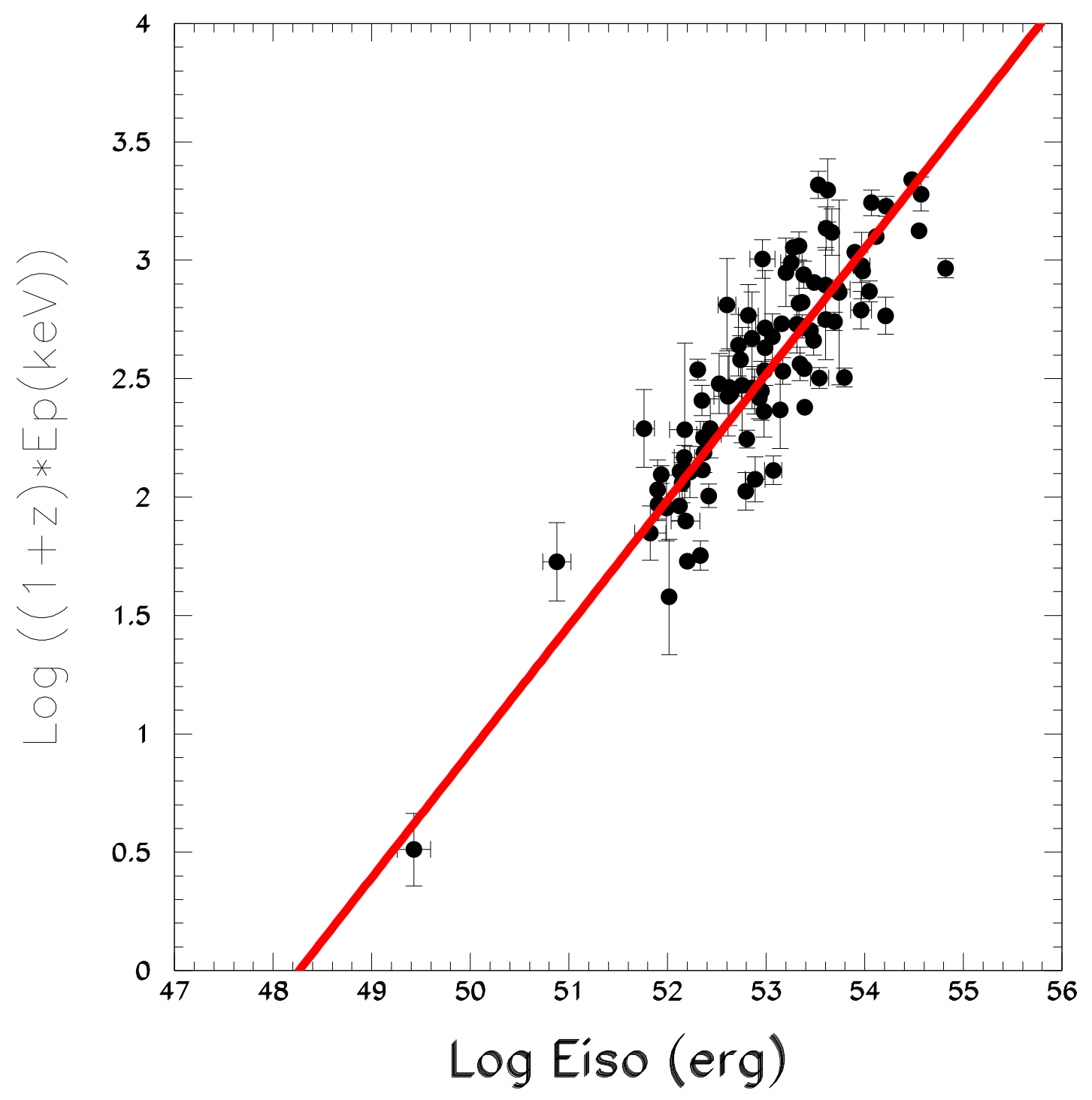

Fig. 1.- The observed correlation between $(1+z) E_{p}$ and $E_{i s o}$ for 121 GRBs with known redshift. The best fit power-law correlation (straight line) has a power-law index 0.54 . 


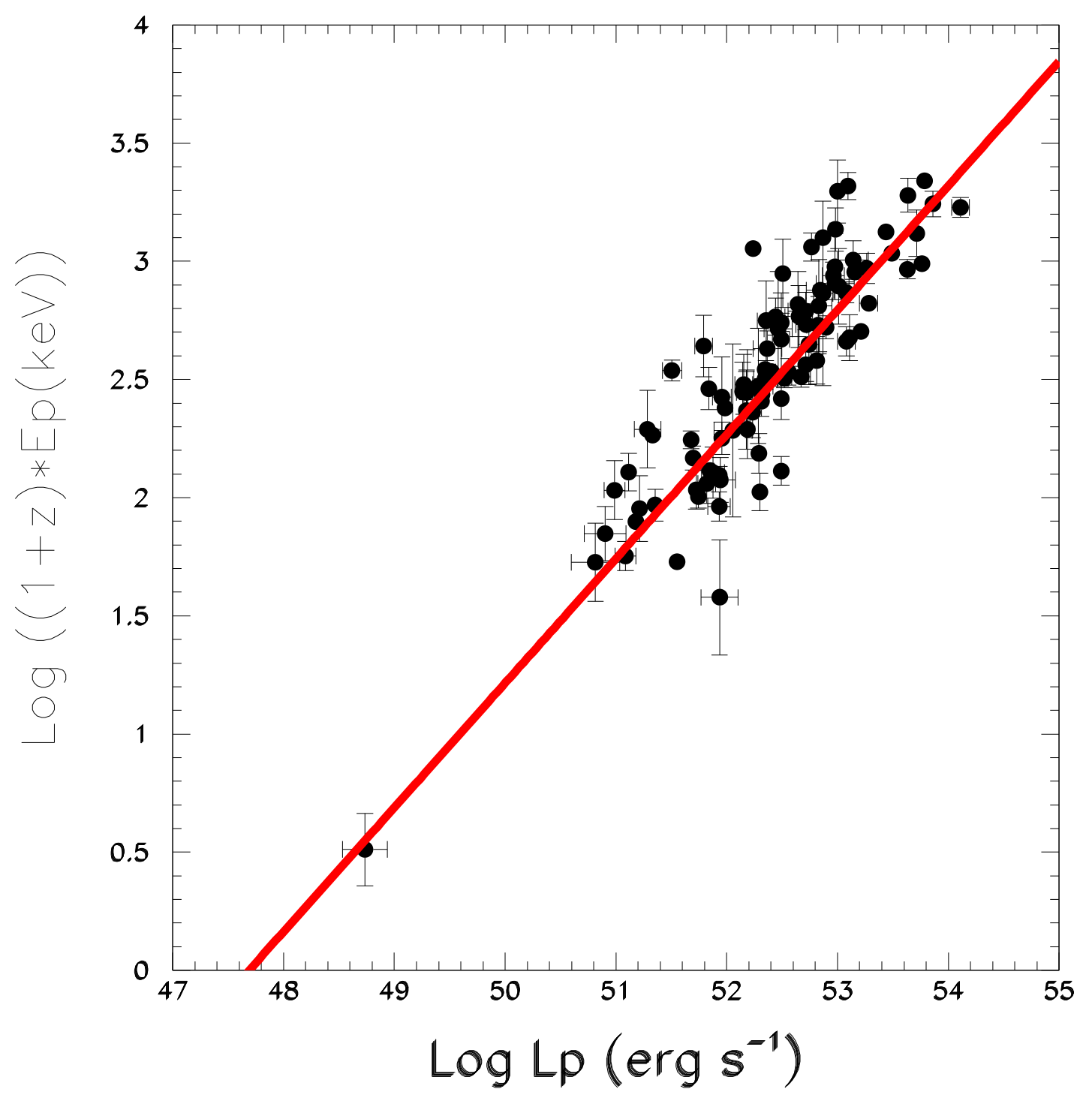

Fig. 2.- The observed correlation between $(1+z) E_{p}$ and $L_{p, s}$ for 121 GRBs with known redshift. The best fit power-law correlation (straight line) has a power-law index 0.526 . 


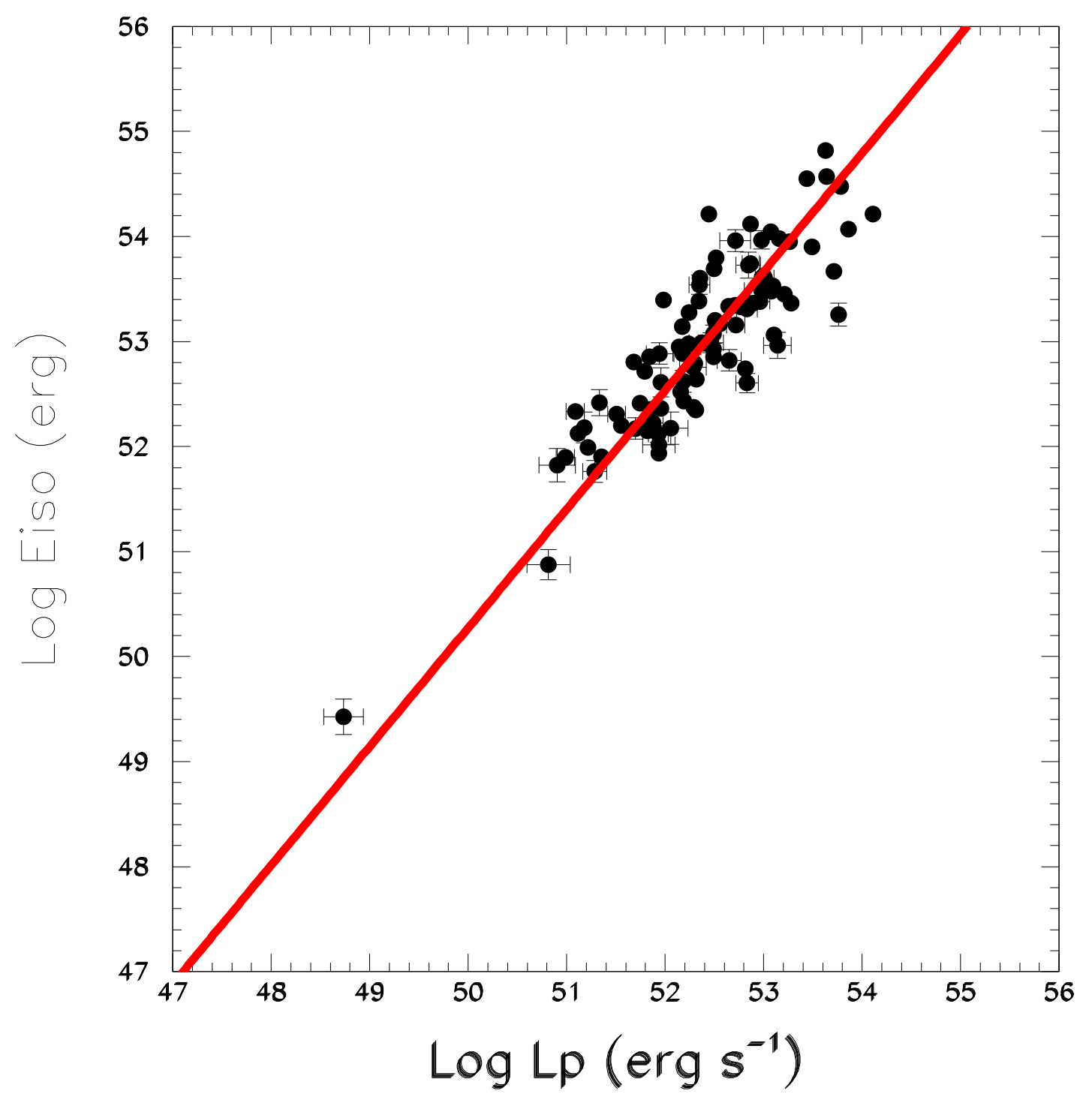

Fig. 3.- The observed correlation between $E_{\gamma, \text { iso }}$ and $L_{p, s}$ for 121 GRBs with known redshift. The best fit power-law correlation (straight line) has a power-law index 1.13. 


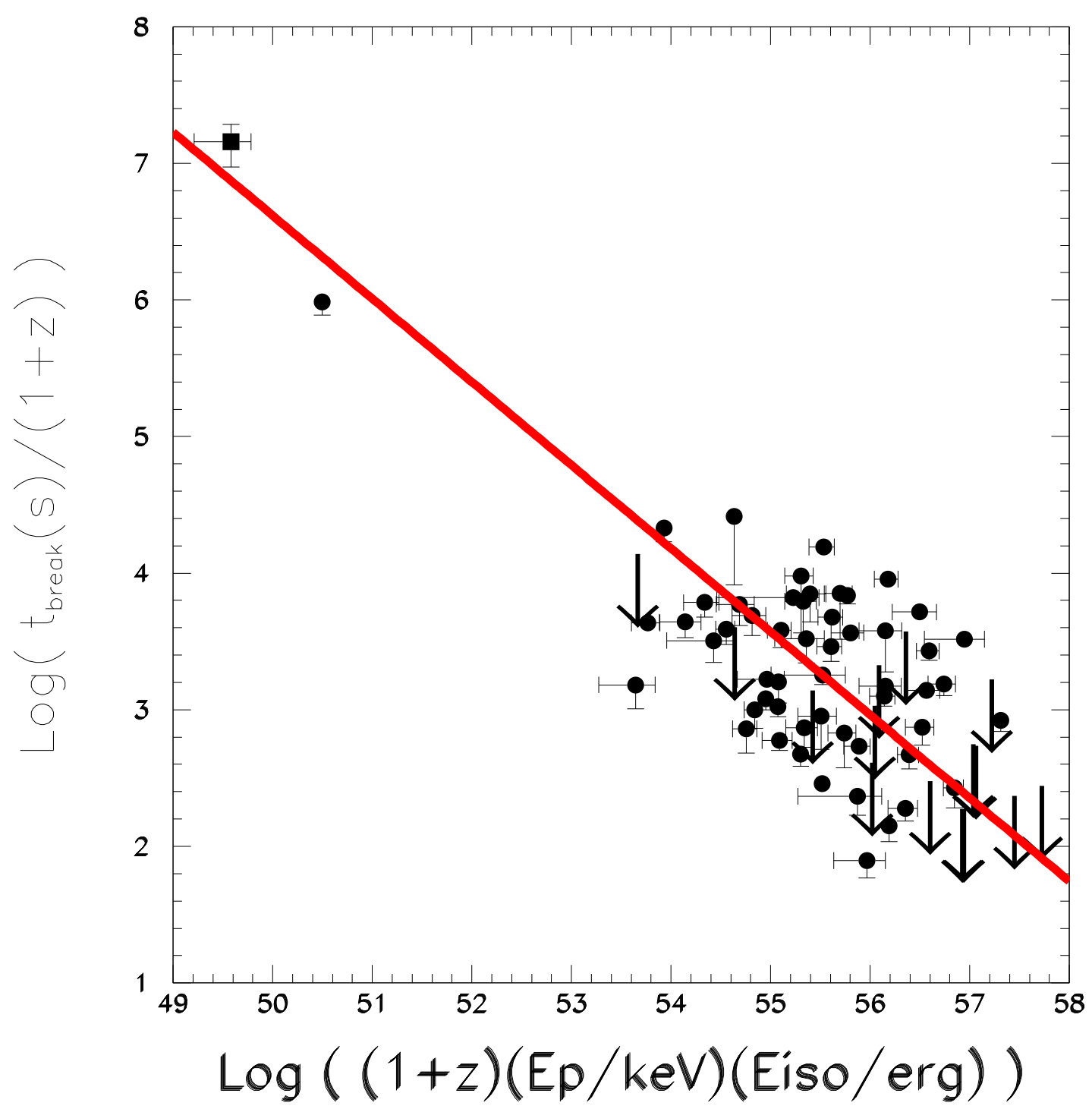

Fig. 4.- The observed triple correlations $t_{b}^{\prime}-E_{p}^{\prime}-E_{i s o}$ in 68 Swift GRBs with measured redshift, $t_{b}^{\prime}, E_{p}^{\prime}$, and $E_{i s o}$ and its best fit power-law (straight line with a power-law index -0.58). Arrows indicate observational upper bounds on early-time deceleration breaks before the beginning of the Swift/XRT observations or hidden under the prompt emission tail. 


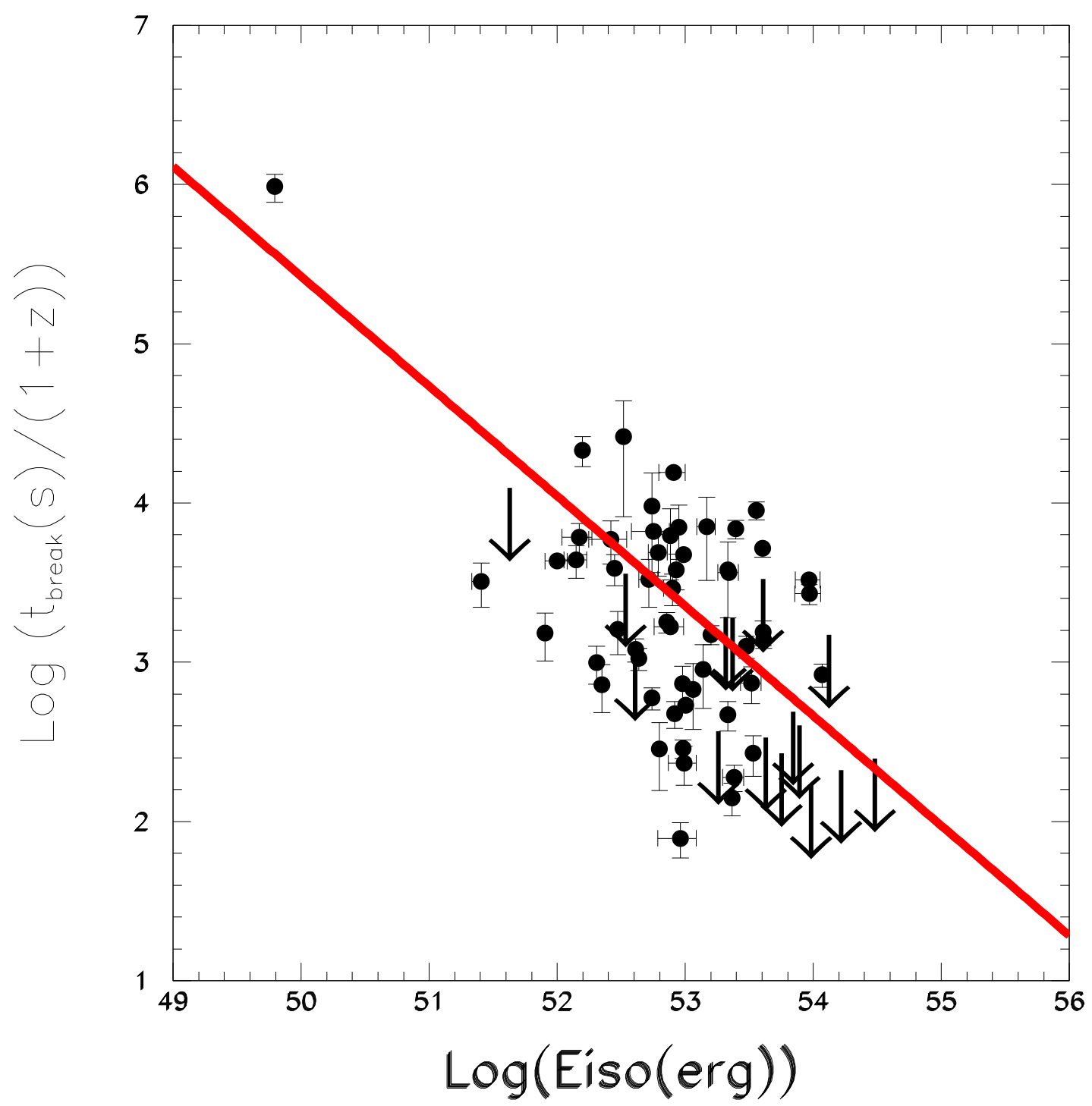

Fig. 5.- The binary correlation $t_{b} /(1+z)-E_{\text {iso }}$ observed in 67 Swift GRBs with measured redshift, $t_{b}^{\prime}$ and $E_{\text {iso }}$ and its best fit power-law (straight line with a power-law index -0.70). Arrows indicate observational upper bounds on early time deceleration breaks before the beginning of the Swift/XRT observations or hidden under the prompt emission tail. 


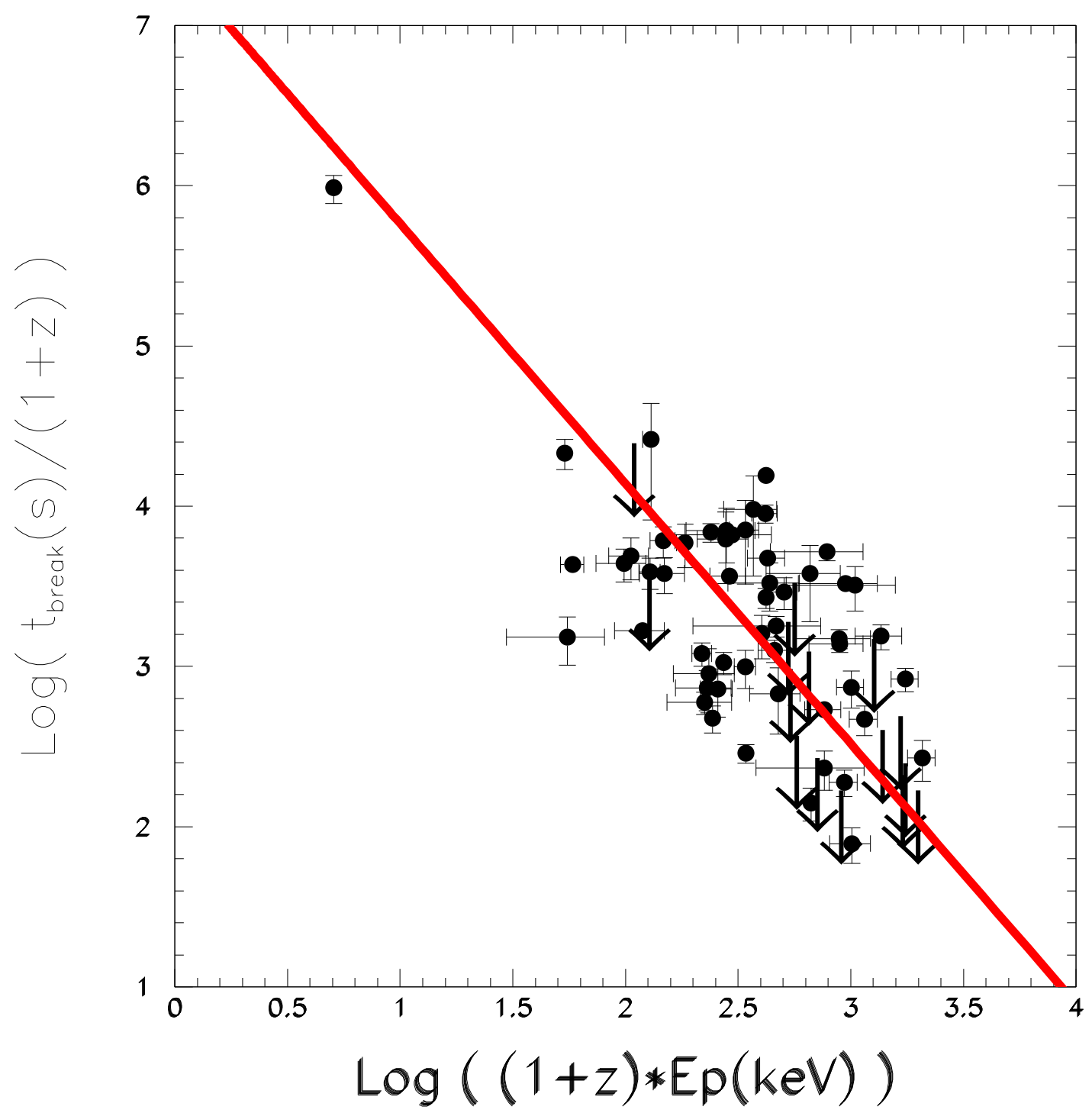

Fig. 6.- The binary correlation $t_{b}^{\prime}-E_{p}^{\prime}$ observed in 67 Swift GRBs with measured redshift, $t_{b}$, and $E_{p}$ and its best fit power-law (straight line with a power-law index -1.61). Arrows indicate observational upper bounds on early time deceleration breaks before the beginning of the Swift/XRT observations or hidden under the prompt emission tail. 